\title{
Circulating microRNAs as Promising Biomarkers in Colorectal Cancer
}

\author{
Óscar Rapado-González 1,2,3, Ana Álvarez-Castro ${ }^{4}$, Rafael López-López 3,5, José Iglesias-Canle 4 , \\ María Mercedes Suárez-Cunqueiro $1,6, *$ (1) and Laura Muinelo-Romay $2,3, *$
}

1 Department of Surgery and Medical Surgical Specialties, Medicine and Dentistry School, University of Santiago de Compostela, 15782 Santiago de Compostela, Spain

2 Liquid Biopsy Analysis Unit, Translational Medical Oncology (Oncomet), Health Research Institute of Santiago (IDIS), 15706 Santiago de Compostela, Spain

3 Instituto de Salud Carlos III, Centro de Investigación Biomédica en Red de Cáncer (CIBERONC), 28029 Madrid, Spain

4 Medical Digestive Service, Complexo Hospitalario Universitario de Santiago de Compostela (SERGAS), 15706 Santiago de Compostela, Spain

5 Translational Medical Oncology (Oncomet), Health Research Foundation Institute of Santiago (IDIS), Complexo Hospitalario Universitario de Santiago de Compostela (SERGAS), 15706 Santiago de Compostela, Spain

6 Oral Sciences Research Group, Health Research Institute of Santiago (IDIS), 15706 Santiago de Compostela, Spain

* Correspondence: mariamercedes.suarez@usc.es (M.M.S.-C.); Laura.muinelo.romay@sergas.es (L.M.-R.); Tel.: +34-881-812-437 (M.M.S.-C.); +34-981-955-073 (L.M.R.)

Received: 15 May 2019; Accepted: 24 June 2019; Published: 27 June 2019

\begin{abstract}
Colorectal cancer (CRC) is one of the most common cancers and a leading cause of cancer-related deaths worldwide. Despite numerous advances in therapeutic approaches, this cancer has a poor prognosis when it is diagnosed at late stages. Therefore, the scientific effort is nowadays directed towards the development of new non-invasive and dynamic biomarkers to improve the survival expectancy of CRC patients. In this sense, deregulated expression of many miRNAs has been shown to play an important role for CRC carcinogenesis and dissemination. Noticeably, an increasing number of studies highlight that circulating miRNAs, including those traveling inside exosomes or those released by tumor cells into circulation, constitute a promising tool for early detection, prognosis and therapy selection of CRC. Therefore, in this review we focus on the clinical potential of blood circulating miRNAs as emerging biomarkers with high value to improve the clinical management of CRC patients, providing a deep and complete perspective of the realities and challenges to translate these biomarkers to the clinical context.
\end{abstract}

Keywords: colorectal cancer; circulating microRNAs; circulating exosomes; tumor biomarkers; liquid biopsy; personalized therapy

\section{Introduction}

Colorectal cancer (CRC) is the third most commonly occurring cancer in men and the second among women and represents the fourth leading cause of cancer-related deaths worldwide [1]. Although CRC incidence and mortality rates vary widely worldwide, its burden is expected to increase by $60 \%$ to more than 2.2 million new cases and 1.1 million cancer deaths by 2030 [2]. The risk of CRC development is associated with numerous factors, including age, family history and inflammatory bowel diseases, diet or lifestyle, and only a small number of cases are due to underlying genetic disorders [3]. Hereditary CRC syndromes such as Lynch syndrome, familial adenomatous polyposis, MUTYH-associated 
polyposis, Peutz-Jeghers syndrome, juvenile polyposis and Cowden/PTEN hamartoma syndrome represent only between $2 \%$ and $5 \%$ of all CRCs [4]. Colorectal carcinogenesis is associated to three important genetic alterations (chromosomal, microsatellite and epigenetic instabilities) which play a key role in the two morphologic multistep pathways: the traditional model adenoma-carcinoma and the serrated neoplasia [5,6]. Importantly, $60-70 \%$ of diagnosed cases in non-screened symptomatic patients are detected in the advanced stage of the disease [7].

Nowadays, several CRC screening modalities are available ranging from non-invasive to invasive tests characterized by specific advantages and disadvantages. Fecal occult blood test and fecal immunochemical test have low sensitivity for detecting pre-neoplastic lesions and a high rate of false positives, while colonoscopy and flexible sigmoidoscopy, which are invasive diagnostic techniques, might be painful and are associated with low adherence rates [8]. To improve CRC, screening different blood-based biomarkers has been investigated as a potential diagnostic tool. Carcinoembryonic antigen (CEA) is a traditional blood-based biomarker widely used for monitoring CRC recurrence but does not represent a suitable diagnostic biomarker due to its low sensitivity and specificity [9]. Importantly, methylated Septin9 assay was approved by the Food and Drug Administration as the first non-invasive blood-based test for CRC screening, however, it has a limited sensitivity to detect adenomas or polyps [10]. Therefore, it is necessary to identify new non-invasive biomarkers with high sensitivity and specificity that could help in early CRC diagnosis, prognosis and treatment.

MicroRNAs (miRNAs or miRs) are a class of small (18-22 nucleotide) non-coding RNAs derived from hairpin precursors that post-transcriptionally regulate gene expression through targeting mRNAs [11]. MiRNAs are known to be involved in a wide variety of biological and pathological process including cancer, having an important role in cell proliferation, migration, invasion and metastasis [12]. In addition, miRNAs can regulate the tumor microenvironment, acting on tumor angiogenesis, immune system and tumor-stromal interactions [12,13]. It is also widely accepted that upregulation of miRNAs entails oncogenesis by downregulating tumor suppressor genes [14]. They modulate specific individual mRNA targets or multiple mRNAs [15] and show aberrant expression patterns in different tumor types [16-18].

Since the discovery of circulating miRNAs, numerous researchers have highlighted their potential as specific biomarkers for cancer. MiRNAs have been detected in various biological fluids including serum, plasma, urine, tears, saliva, seminal fluid, cerebrospinal fluid, extracellular fluid, and others [19]. Circulating miRNAs show a remarkable stability because they are protected against endogenous RNAses by different protective mechanisms such as miRNA modifications, miRNAs encapsulation by membranous extracellular vesicles or miRNA-binding proteins as Argonaute 2 (Ago2) or high-density lipoproteins [20]. Three major pathways could explain the presence of miRNAs in body fluids: (1) passive leakage of cellular miRNAs into the circulation due to cell apoptosis and tissue damage, (2) active selective secretion as microvesicles-free miRNAs (cell-free miRNAs) and (3) active secretion via binding to cell-derived microvesicles and exosomes [21]. Therefore, the expression profile of circulating miRNAs may reflect the miRNA profile of primary tumor or metastatic lesions, facilitating the detection of the tumor at different stages in a non-invasive manner. This approach represents a unique opportunity to discover novel biomarkers involved in CRC carcinogenesis. Considering the potential of miRNAs as valuable CRC biomarkers, we provide an overview of scientific evidence of circulating miRNAs for diagnosis, prognosis and therapy prediction in CRC patients (Figure 1 and Table S1). 


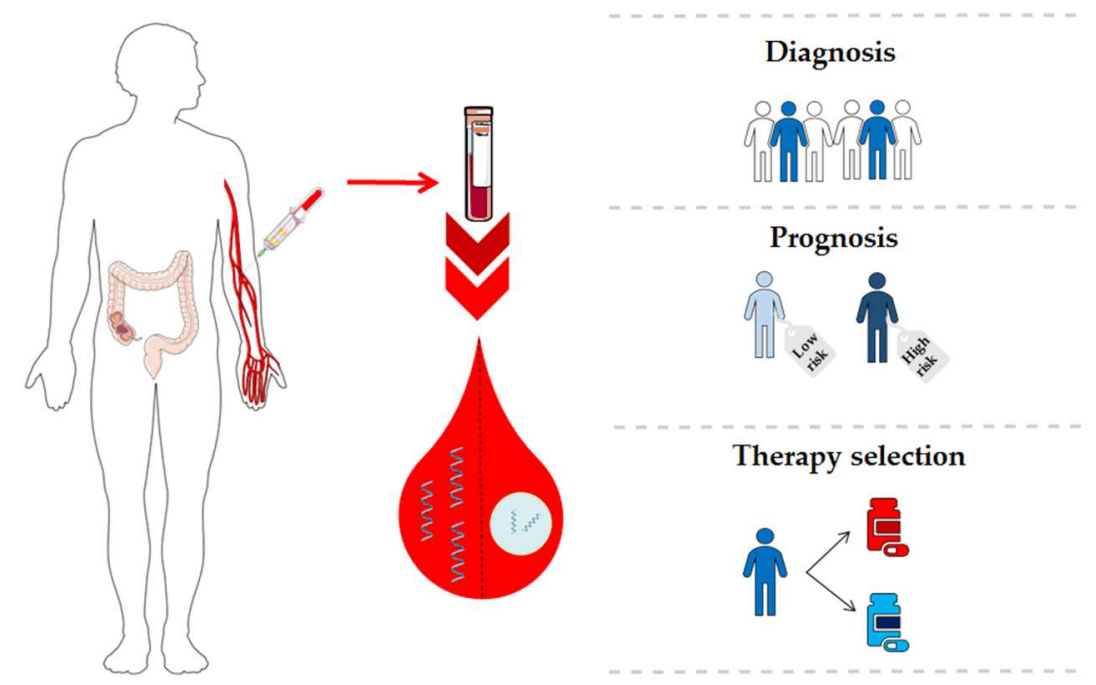

Figure 1. Clinical applications of circulating microRNAs (miRNAs) in colorectal cancer (CRC).

\section{MiRNAs as Diagnostic Biomarkers in CRC}

The first report that assessed the use of circulating miRNAs as a non-invasive diagnostic test for CRC was performed in 2009 [22]. Since then, several researchers have focused on the diagnostic value of circulating miRNAs for CRC detection [23,24]. Furthermore, ongoing research is aimed to identify specific miRNA profiles for early and advanced CRC stages, and even determine if there is a correlation with the tumor-node-metastasis (TNM) staging system. The discovery of miRNA signature involved in each step of the disease will increase the diagnostic accuracy in CRC. Therefore, circulating miRNAs could be potential non-invasive biomarkers for CRC diagnosis (Figure 2 and Table S2).

\subsection{Cell-Free miRNAs for Early CRC Diagnosis}

Detection of precancerous lesions and early-stage CRC (I-II) is essential to reduce mortality. In the last few years, several studies [25-28] have evaluated the potential of circulating miRNAs as diagnostic biomarkers in CRC. Huang et al. were the first to evaluate the diagnostic value of plasma miRNAs for early CRC detection. They found significant elevated expression of miR-29a and miR-92a in 37 patients with advanced adenomas in comparison to healthy controls. Receiver operating characteristic (ROC) curve analysis yielded an area under the curve (AUC) of 0.769 for miR-29a with $62.2 \%$ sensitivity and $84.7 \%$ specificity, and an AUC of 0.749 for miR-92a with $64.9 \%$ sensitivity and $81.4 \%$ specificity. However, when both miRNAs were combined, an increase of the AUC value was observed [25]. Further research has revealed different miRNAs as potential biomarkers for early CRC diagnosis in serum and plasma. Wang et al. profiled 86 differentially expressed miRNAs in CRC patients and healthy controls, choosing 17 novel miRNAs with fold changes $>5$ for further validation. Plasma expression levels of miR-601 and miR-760 were significantly decreased in CRC compared to controls, showing at early stages a remarkedly decreased expression in respect to advanced adenomas and healthy controls. In addition, miR-601 and miR-760 expression was significantly reduced in advanced adenomas compared to healthy controls and increased compared to CRC. Interestingly, the combination of these biomarkers and CEA yielded an AUC of 0.805 and $80.4 \%$ sensitivity and $65.5 \%$ specificity, indicating their potential diagnostic value for early CRC [26]. Later, Kanaan et al. reported a panel of eight upregulated cell-free miRNAs (miR-532-3p, miR-331, miR-195, miR-17, miR-142-3p, miR-15b, miR-532 and miR-652) that differentiated advanced adenomas from healthy controls with $88 \%$ sensitivity and $64 \%$ specificity. Moreover, a 5-miRNA panel (miR-331, miR-15b, miR-21, miR-142-3p and miR-339-3p) distinguished patients with advanced adenomas from CRC with an AUC of 0.856 and $91 \%$ sensitivity and $69 \%$ specificity [29]. These findings suggest the existence of different miRNA profiles in the early steps of CRC carcinogenesis. In another study, miR-21, miR-29a and miR-125b showed significantly higher 
serum expression levels in patients with early colorectal neoplasia compared to healthy controls, showing AUC values of 0.706 for miR-21, 0.741 for miR-29a and 0.806 for miR-125b. Also, miR-21, miR-29a and miR-125b could discriminate patients with advanced neoplasia from non-advanced neoplasia and healthy controls showing AUC values that ranged from 0.690 to 0.731 . Interestingly, the combination of these three miRNAs improved the discriminatory power for both early (AUC $=0.826$ ) and advanced (AUC $=0.759$ ) colorectal neoplasms. These three miRNAs were significantly increased in patients with tubular adenomas and high-grade intraepithelial neoplasms while miR-29a and miR-125b were also significantly elevated in tubule-villous adenomas and small colorectal neoplasms $(\leq 5 \mathrm{~mm})$ [27].

\section{Circulating miRNAs for CRC diagnosis}

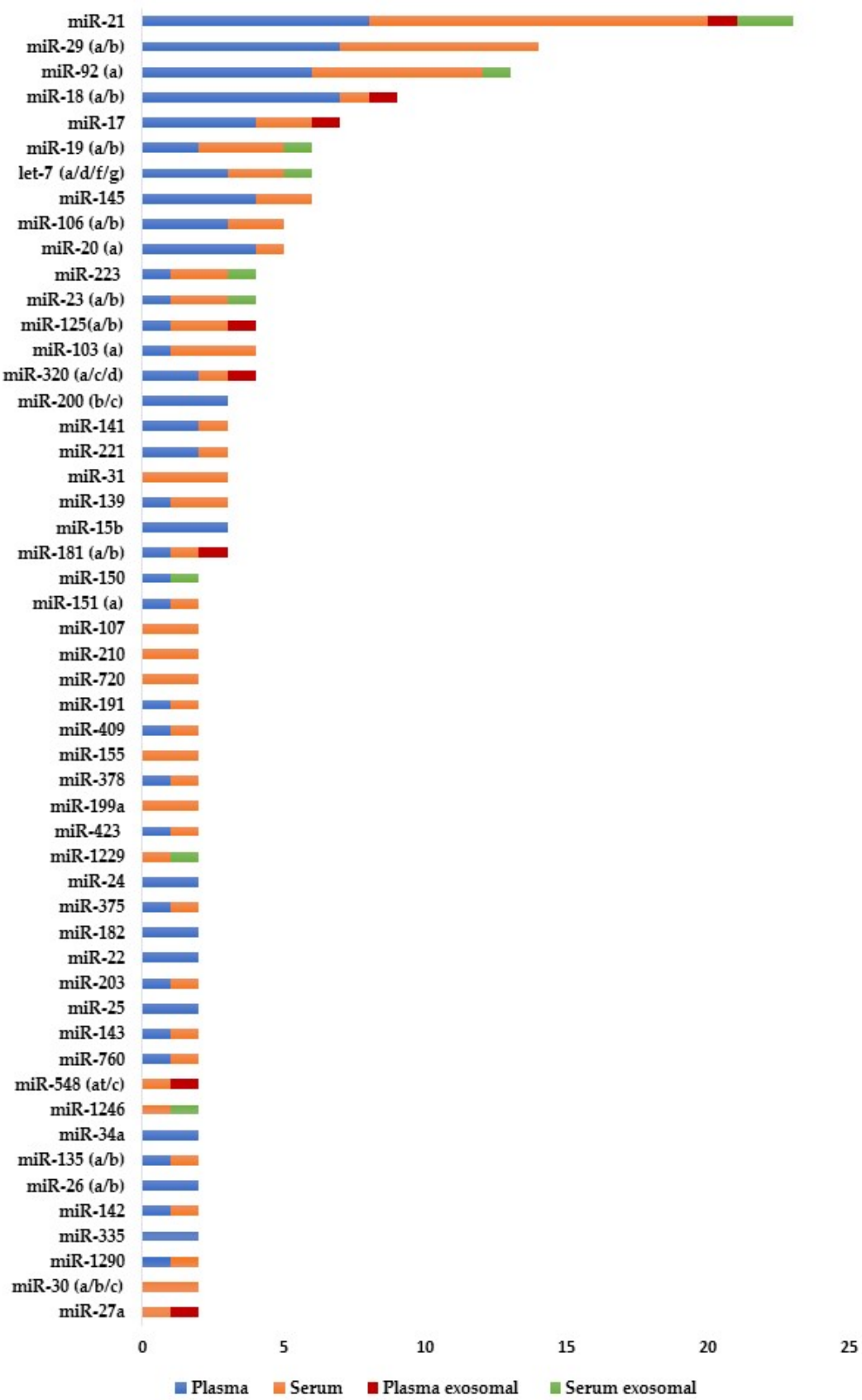

Figure 2. List of circulating miRNAs reported in two or more studies for CRC diagnosis. These miRNAs were significantly deregulated in CRC compared to healthy individuals $(p<0.05)$. References are provided in Table S2. 
In addition to these studies, massive characterization of cell-free miRNAs between CRC patients and healthy individuals provided the researchers alternative miRNAs panels for early CRC detection. Thus, Hofsli et al. developed a model of 21 miRNAs that showed the same expression profile at early and advanced CRC stages. This serum-based miRNA model discriminated early CRC from healthy controls with $90 \%$ specificity and $87.5 \%$ sensitivity [30]. Wang et al. also identified a 3-miRNA panel (miR-7, miR-409-3p and miR-93) with high diagnostic accuracy for discriminating early CRC from healthy individuals $(82 \%$ sensitivity and $89 \%$ specificity, AUC $=0.892)$ [28]. Using next generation sequencing, Vychytilova-Faltejskova et al. found 54 miRNAs significantly deregulated in the serum of 144 CRC patients and 96 healthy controls. Of these, 4 miRNAs (miR-23a-3p, miR-27a-3p, miR-142-5p and $\mathrm{miR}-376 \mathrm{c}-3 \mathrm{p}$ ) were validated as diagnosis biomarkers demonstrating a high discriminatory power to detect early CRC $(81 \%$ sensitivity and specificity, $\mathrm{AUC}=0.877)$ [31]. More recently, a plasma 6-miRNA panel was validated for early CRC detection in a multicenter study including 96 CRC, 101 advanced adenomas and 100 healthy individuals. This miRNA signature demonstrated $95 \%$ sensitivity and $90 \%$ specificity for advanced adenomas and $94 \%$ sensitivity and $87 \%$ specificity for early CRC [32]. Furthermore, other miRNAs, such as miR-506, miR-4316, miR-182 and miR-30a-5p have also been evaluated as potential markers for early CRC detection [33-35].

Overall, all these studies combining different panels of cell-free miRNAs, reach high accuracy to detect the presence of CRC at early stages with rates of sensitivity and specificity above $80 \%$, making evident the great potential of these molecules as screening biomarkers.

\subsection{Cell-Free miRNAs for Advanced CRC Diagnosis}

As previously mentioned, miRNAs have been involved in the advance of $\mathrm{CRC}$, promoting invasion, migration and the progression of the disease through epithelial to mesenchymal transition (EMT) activation and other mechanisms behind the tumor spread [36]. Accordingly, researchers have examined the expression levels of circulating miRNAs in advanced stages and their diagnostic value for discriminating patients with lymph nodes and/or distant metastasis. In 2011, Cheng et al. analyzed 3 miRNAs (miR-21, miR-92a and miR-141) in the plasma of CRC patients, finding significantly higher levels of miR-141 in stage IV CRC patients compared to healthy individuals. Plasma miR-141 was significantly correlated with tumor stages and upregulated in distant compared to non-distant metastasis. Thus, ROC curve analysis showed that miR-141 discriminated stage IV from stages I-II with $66.7 \%$ sensitivity and $80.8 \%$ specificity, stage IV from stage III with $66.7 \%$ sensitivity and $89.7 \%$ specificity, and stage IV from stages I-III with $66.7 \%$ sensitivity and $84 \%$ specificity. In addition, the combination of miR-141 and CEA could detect additional metastatic CRC patients which were not detected using one of both biomarkers independently [37]. These results indicate that plasmatic miR-141 levels represent a non-invasive biomarker for detecting metastatic CRC patients. In this line, Wang and $\mathrm{Gu}$ evaluated the expression levels of miR-29a, miR-17-3p and miR-92a in serum of 38 metastatic CRC patients compared to 36 non-metastatic CRC cases. They found that serum miR-29a was significantly upregulated in patients with liver metastasis showing $75 \%$ sensitivity and specificity (AUC of 0.803 ) for discriminating metastatic from non-metastatic patients. They also observed a significant positive correlation between serum and tissue miR-29a expression levels in metastatic CRC patients, with its levels associated with advanced T stages [38]. Other studies have described upregulated levels of miR-126, miR-141, miR-21 and miR-200 in CRC patients with liver affectation [39,40]. Some of these miRNAs were associated with tumor metastasis through EMT regulation targeting ZEB1 and ZEB2 transcription factors [41]. Serum miR-885-5p high levels were also significantly associated with high TNM stage, lymph node and distant metastasis [42]. Recently, also upregulated serum levels of miR-103 were associated with lymph node metastasis and advanced tumor stage, reflecting its involvement in the progression of CRC [43]. Other circulating miRNAs expression patterns have been identified as potential biomarkers in CRC advanced stages. Giráldez et al. reported a plasma 3-miRNA panel (miR-19a, miR-19b and miR-15b) with high discriminatory power to detect advanced stages from healthy individuals with $76.19 \%$ sensitivity and $77.36 \%$ specificity (AUC $=0.81$ ) [44]. In 
a similar study, Brunet-Vega et al. analyzed 11 upregulated tissue miRNAs in serum samples from 30 stage III CRC patients and 26 healthy individuals, finding only significantly increased expression of miR-18a and miR-29a in the patients' cohort [45]. These results suggest the potential role of these miRNAs in the development of lymph node metastasis. Another miRNA analysis performed in 187 CRC patients and 47 healthy controls revealed significantly increased miR-141, let-7f-2, miR-628-5p, miR-203 and miR-200b expression levels in advanced stages compared to early stages and healthy individuals. In addition, miR-15b, miR-526, miR-96, miR-148a and miR-22 were also significantly upregulated in advanced stages compared to healthy individuals. Logistic regression analysis showed that plasma miR-203 differentiated advanced stages from early stages with $74.7 \%$ sensitivity and $71.4 \%$ specificity and, again, miR-141 distinguished stage IV from stages I-III with $80.0 \%$ sensitivity and $86.1 \%$ specificity [46]. Finally, downregulation of specific miRNAs was also described in advanced disease. Thus, serum levels of miR-139-3p were significantly downregulated in advanced stages CRC patients in respect to controls, showing $97.2 \%$ sensitivity and $97.8 \%$ specificity to identify the patients and demonstrating higher diagnostic accuracy compared to blood CEA levels [47].

\subsection{Exosomal miRNAs for CRC Diagnosis}

Nowadays, exosomal miRNAs have emerged as promising diagnostic biomarkers in cancer. Thus, Ogata-Kawata et al. identified 16 miRNAs significantly upregulated in serum exosomes from CRC patients compared to healthy controls as well as in colon cancer cell lines using a microarray-based approach. From those, serum exosomal let-7a, miR-1224-5p, miR-1229, miR-1246, miR-150, miR-21, miR-223 and miR-23a showed significantly lower levels after surgical resection of the primary tumor demonstrating the tumoral origin of these exosomal miRNAs. According to ROC curve analysis, miR-1246 and miR-23a showed sensitivities of $95.5 \%$ and $92 \%$ respectively, reflecting the high diagnostic power of both miRNAs. Notably, stage I patients showed significantly increased levels of seven miRNAs (let-7a, miR-1229, miR-1246, miR-150, miR-21, miR-223, and miR-23a), suggesting a role of exosomal miRNAs in the early steps of CRC development [48]. Wang et al. analyzed nine plasmatic exosomal miRNAs from 50 early CRC patients and matched healthy individuals but only miR-125-3p and miRNA-320c were significantly upregulated in CRC compared to healthy individuals, showing AUC values of 0.6849 and 0.5982 , respectively. Interestingly, an important increase of diagnostic power for early CRC stage was observed by combining CEA and miR-125-3p (AUC $=0.8552)$ [49]. These data support evidence about the possibility to combine exosomal miRNAs and traditional tumor markers as useful CRC screening methods. MiR-21 has been also described as upregulated in plasmatic exosomes from CRC patients compared to healthy individuals. Moreover, miR-21 was higher in advanced respect to early stages and also in patients with liver metastasis [50]. Another circulating exosomal profile study including 77 CRC patients and 20 healthy individuals identified 7 deregulated miRNAs of which miR-638, miR-5787, miR-8075, miR-6869-5p and miR-548c-5p were significantly downregulated and miR-486-5p and miR-3180-5p were significantly upregulated. Among these, low expression of miR-638 was associated with a higher risk of liver metastasis and advanced TNM stage. Furthermore, a network analysis showed that miR-638, miR-5787, miR-8075, miR-6869-5p, and miR-548c-5p may regulate the glucose metabolism in CRC [51]. Recently, miR-17-5p, miR-181a-5p, miR-18a-5p and miR-18b-5p, which were detected previously as cell-free miRNAs in plasma, were described as upregulated in plasmatic exosomes. These findings suggest that circulating miRNAs are present in the bloodstream both bound to Ago2, as normally happens in cell-free miRNAs, or encapsulated in exosomes [52]. Also, exosomal miR-27a and miR-130a showed higher levels in plasma from CRC patients compared to healthy individuals and adenoma patients, providing the combination of both miRNAs a high diagnostic accuracy (AUC $=0.801$ ) [53]. However, further research should be performed to reveal the mechanisms through which tumor cells secreted exosomes containing specific miRNAs in order to improve its clinical application as biomarkers and potential therapeutic targets. 


\section{3. miRNAs as Prognostic Biomarkers in CRC}

The first report that established a relationship between circulating miRNAs and prognosis in CRC was performed in 2010 [54]. Since then, several promising circulating miRNAs were associated with poor progression-free survival (PFS) and overall survival (OS) times in CRC patients. Some of the miRNAs that have demonstrated diagnostic potential were also identified as useful prognostic biomarkers, while other miRNAs have been exclusively associated with CRC prognosis. Therefore, circulating miRNAs could be potential non-invasive biomarkers for CRC prognosis (Figure 3 and Table S3).

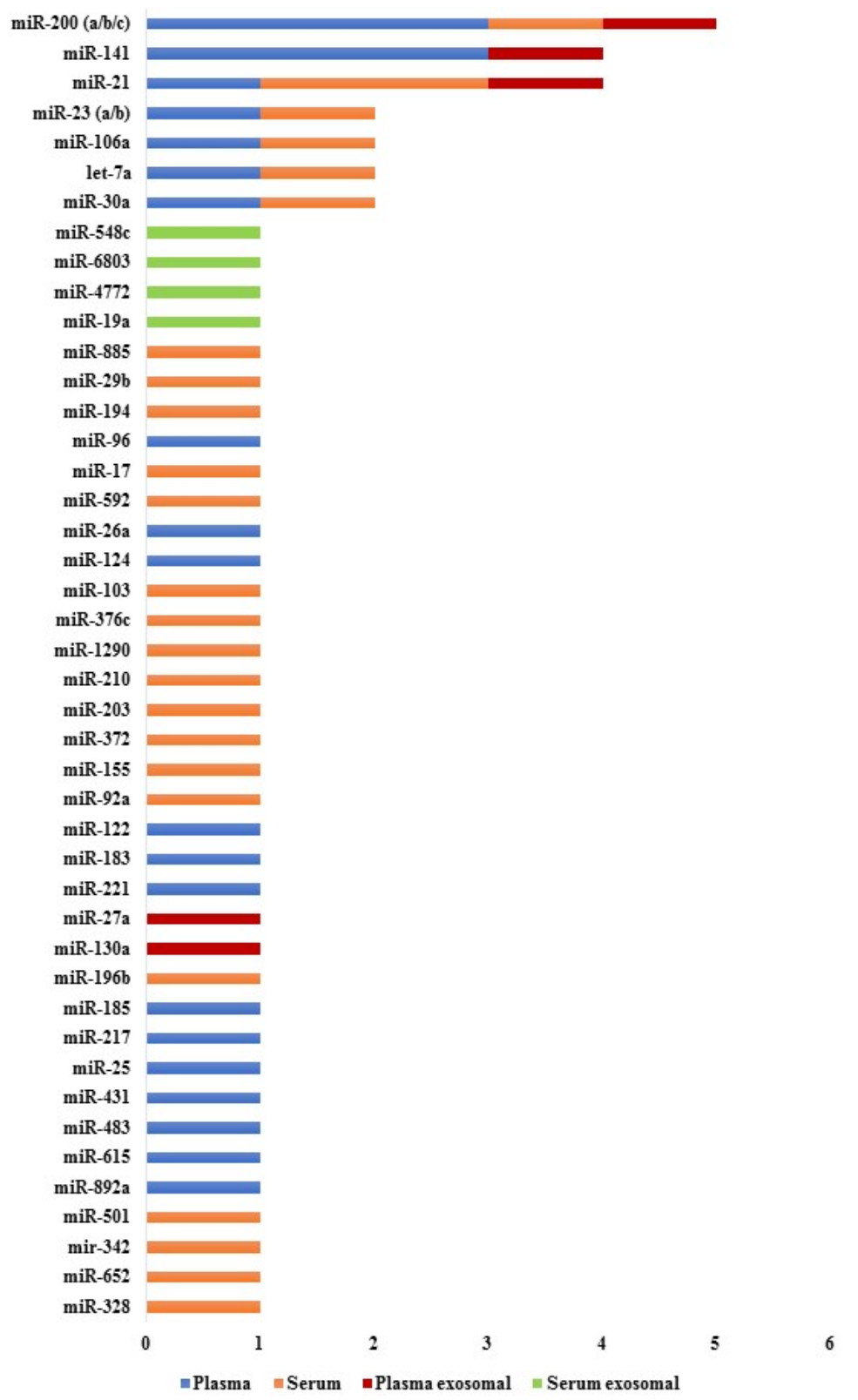

Figure 3. List of circulating miRNAs reported in one or more studies for CRC prognosis. These miRNAs were significantly associated with survival $(p<0.05)$. References are provided in Table S3.

\subsection{Cell-Free miRNAs for CRC Prognosis}

Circulating miRNAs that provide information about biological behavior and clinical evolution of CRC are good candidates for predicting disease prognosis. Pu et al. analyzed plasmatic levels of miR-21, miR-221 and miR-222 in 103 CRC patients and its correlation with p53, CEA, estrogen and 
progesterone receptors status. In this study, factors known as histological grade, clinical stage, presence of metastasis and high levels of miR-221 were significantly correlated with poor OS. Interestingly, a significant correlation between plasma miR-221 level and p53 protein expression was found but the role of miR-221 in the regulation of p53 is nowadays unknown [54]. Further, several works have found high levels of miR-17-3p [55], miR-106a [55], miR-21 [56], miR-92a [57], miR-1290 [58], miR-210 [59], miR-183 [60], miR-885-5p [42], miR-592 [61], miR-196b [62] or miR-155 [63] associated with lower PFS or OS. More recently, serum miR-203 has been proposed as another promising prognostic and metastasis-predictive biomarker in CRC. High circulating miR-203 levels were associated with high TNM stage, presence of lymph node infiltration, distant and peritoneal metastasis, and a shorter OS. ROC curve analysis showed that miR-203 could discriminate, with $62.5 \%$ sensitivity and $77.68 \%$ specificity, patients with poor prognosis. In addition, miR-203 was an independent biomarker for predicting the formation of lymph node, liver and peritoneal metastasis [64].

The prognostic impact of miRNA-200 family has been described in several studies focused on CRC [37,65-67]. Chen et al. showed that high plasma miR-141 levels were significantly associated with a poor OS in metastatic CRC patients [37]. Later, Toiyama et al. analyzed the expression levels of miR-200b, miR-200c, miR-141 and miR-429 in CRC serum samples, and they observed significantly elevated levels of miR-200c in stage IV tumors compared to stages I-III and healthy controls. Interestingly, high serum miR-200c levels were significantly associated with lymph node affectation and liver metastasis. Furthermore, multivariate logistic regression analysis showed that miR-200c was an independent prognostic marker for predicting shorter OS and tumor recurrence in stages II and III [67]. In another study, Maierthaler et al. performed a miRNA profiling in 20 metastatic and 20 non-metastatic patients, including in each group 10 patients with "good prognosis" and 10 patients with "bad prognosis". A total of 11 miRNAs were validated in a large cohort of patients, finding significantly increased levels of miR-122, miR-141, miR-200a, miR-200b and miR-203a in metastatic compared to non-metastatic patients. According to univariate and multivariate analysis, "bad prognosis" patients showed significantly higher levels of miR-122 in the metastatic CRC group and in the non-metastatic CRC group significantly low levels of miR-200c were observed suggesting a different behavior of cell-free miRNAs during the tumor evolution. Further, high expression levels of miR-200a were only correlated with a shorter OS in metastatic CRC. Finally, high levels of miR-122 were associated with a shorter OS and relapse-free survival in both metastatic and non-metastatic CRC [65]. More recently, high levels of miR-200c and miR-141 in plasma obtained after mesenteric vein blood collection were associated with shorter OS in CRC. By contrary, these findings were not observed in blood from peripheral veins, which reflects a major potential for detecting tumor derived biomarkers from tumor-draining veins [68].

It is noteworthy to point out that downregulated miRNAs have also been associated with an unfavorable prognosis in CRC. Low levels of miR-30a-5p, miR-194, miR-29b and miR-23b were associated with a shorter PFS and OS [35,69,70]. These miRNAs were significantly associated with a high TNM stage. In particular, miR-23b was significantly associated with tumor depth, distant metastasis and tumor recurrence [70]. Currently, it is well known that the combination of various miRNAs with clinical data improves not only the ability to diagnose but also the prognosis. In fact, Vychytilova-Faltejskova et al. designed a signature for prediction of three-year OS consisting of three clinical factors (age, gender and stage) and two miRNAs (miR-23a-3p, miR-376c-3p). This model accurately predicted the outcome of more than $70 \%$ of CRC patients, even for each TNM stage independently [31]. Recently, Vafaee et al. developed a network based on an algorithm to discover robust miRNA signatures providing information about their biological role in CRC progression. By this approach, a plasma 11-miRNA signature was associated to several pathways related with CRC prognosis [71]. 


\subsection{Exosomal miRNAs for CRC Prognosis}

Several circulating exosomal miRNAs have been reported as potential prognosis biomarkers for CRC patients. Matsumura et al. identified six serum exosomal miRNAs associated with liver CRC metastasis after a global microarray profiling in 124 CRC patients. In this study, upregulated expression of miR-19a and miR-92a was observed in CRC patients compared to healthy controls, with high levels of exosomal miR-19a significantly associated with tumor infiltration, lymph node and liver metastasis and, therefore, with a higher risk of tumor recurrence and a worse overall prognosis [72]. Liu et al. validated as prognosis markers a total of 10 serum exosomal miRNAs identified by RNA sequencing in 84 samples of patients with stages II and III CRC collected after tumor resection and before adjuvant therapy. Downregulated levels of miR-4772-3p and upregulated levels of miR-4732-5p were observed in recurrent patients compared to the non-recurrent group. ROC curves demonstrated that miR-4772-3p allowed to discriminate recurrent from non-recurrent patients with $78.6 \%$ sensitivity and $77.1 \%$ specificity, which evidenced its potential as a prognostic biomarker for tumor recurrence [73]. Interestingly, Tsukamoto et al. evaluated the prognostic value of plasma exosomal miR-21 according to TNM classification. Among CRC patients, high miR-21 expression levels were significantly associated with a poor prognosis, determining a significantly worse disease-free survival (DFS) and OS in stages II-III CRC, while in stage IV only the OS was significantly worse in patients with high exosome-derived miR-21. Importantly, the multivariate analysis revealed that miR-21 was an independent prognostic biomarker for DFS and OS in stages II-III and for OS in stage IV [50]. Similarly, high serum exosomal miR-6803-5p levels were significantly and independently associated with shorter DFS and OS in CRC patients, with this predictive value higher in patients with advanced stages and liver metastasis [74]. Other exosome-encapsulated miRNAs associated with a poor prognosis in CRC were miR-548c-5p, miR-27a and miR-130a [53,75]. In particular, decreased expression levels of miR-548c-5p were significantly associated with a shorter OS, with this downregulation more significant in patients with liver metastasis and advanced tumor stages. Then, multivariate analysis demonstrated that low levels of miR-548c-5p were independently associated with poor OS. Interestingly, potential miR-548c-5p target genes, such as STAT3, PTPRO, IRF, interleukins and their receptors, implicated in CRC carcinogenesis and dissemination have been described providing valuable information regarding the role of this miRNA in the context of CRC [75]. Recently, Fu et al. carried out an exosomal miRNA sequencing from primary (SW480) and lymph node metastatic (SW620) CRC cell lines. Expression levels of 11 deregulated miRNAs (miR-17, miR-19a, miR-20, miR-92a, miR-7, miR-181a, miR-375, miR-194, miR-30d, miR-192, and miR-146) were further validated in serum exosomes from 29 CRC patients and 11 healthy individuals, finding significantly increased levels of miR-17 and miR-92a in metastatic CRC compared to controls. In addition, circulating exosomal levels of both miRNAs were significantly correlated with pathological stages and grades of CRC, suggesting their potential as prognostic biomarkers for primary and metastatic CRC [76].

\section{4. miRNAs as Therapy Prediction Biomarkers in CRC}

Chen et al. were the first researchers that explored the role of circulating miRNAs as non-invasive biomarkers for predicting the development of resistance to chemotherapy in CRC [77]. Due to cancer patients frequently showing resistance to therapy during the course of the disease, there is an urgent need to find precise predictive biomarkers that choose effective drugs for each patient and identify early therapy resistance to avoid overtreatments with the consequent toxic effects.

\subsection{Cell-Free miRNAs for CRC Therapy Prediction}

Over the last few years, several studies [77-79] have focused on plasma and serum miRNAs as potential biomarkers to predict the sensitivity of CRC to chemotherapy. Chen et al. characterized serum samples from 16 responders and non-responders CRC patients under FOLFOX based chemotherapy using microarrays. They found five differentially expressed miRNAs (miR-221, miR-222, miR-122, 
miR-19a, miR-14) that were further validated in 72 CRC patients. From them, only serum miR-19a was significantly upregulated in non-responder patients, being able to discriminate non-responder from responder patients with $66.7 \%$ sensitivity and $63.9 \%$ specificity [77]. Similarly, Zhang et al. performed a genome-wide expression profiling to identify predictive serum miRNAs for oxaliplatin-based chemotherapy. After this global gene expression analysis, the authors found five miRNAs (miR-20a, miR-130, miR-145, miR-216 and miR-372) significantly downregulated in a large-scale validation phase, including 93 responder and 80 non-responder patients. This 5-miRNA panel showed $92 \%$ sensitivity and $88 \%$ specificity to discriminate primary sensitive and resistant patients, demonstrating the potential of this panel as a predictive tool for guiding CRC therapy selection [78]. In another study, Kjersem et al. evaluated the expression of 742 plasma miRNAs in metastatic CRC patients before and after chemotherapy. Before onset treatment, the upregulation of three miRNAs (miR-106a, miR-130b and miR-484) was associated with a lack of response to 5-fluorouracil and oxaliplatin but not with a reduced survival. In addition, high plasma levels of miR-148 and miR-27b were associated with a shorter PFS and high expression of miR-326 was associated with shorter PFS and OS [79]. Recently, Ji et al. discovered a serum 4-miRNA signature (miR-328-3p, miR-652-3p, miR-342-3p and miR-501-3p) significantly correlated with therapeutic outcome in stage II-III CRC patients who were treated with fluorouracil-based adjuvant chemotherapy with or without leucovorin, levamisole or oxaliplatin. In both stages, a high-risk signature score was significantly associated with a poor DFS and OS, indicating the potential of this miRNA signature as an independent predictor of the response to adjuvant chemotherapy [80].

Interestingly, miRNAs have been also involved in angiogenic signaling and vascular integrity [81]. Hansen et al. analyzed the predictive value of miR-126 in 68 CRC patients treated with first-line chemotherapy combined with bevacizumab. During treatment, upregulated levels of plasma miR-126 were related with a lack of response to the anti-angiogenic therapy. Also, changes in tumor size were significantly correlated with the plasmatic levels of miR-126. Further, survival analysis revealed favorable prognosis in those patients with high miR-126 levels at baseline and a decrease of these levels during the treatment [82]. Similarly, Ulivi et al. analyzed a panel of plasmatic miRNAs involved in the angiogenic process with value as biomarkers to predict bevacizumab efficacy in advanced CRC. High baseline levels of miR-20b-5p, miR-29b-3p and miR-155-5p were significantly associated with better PFS and OS rates. Importantly, an increase of $\geq 30 \%$ of miR-155-5p after one month of therapy was significantly associated with shorter PFS and OS, indicating its utility for the treatment monitoring. The authors of this study proposed these three miRNAs as potentially involved in the angiogenic regulation, acting as predictive markers to select bevacizumab treatment in metastatic CRC patients [83]. In addition, high circulating miR-345 levels were associated with a lack of response to cetuximab and irinotecan with an odds ratio of 5.37 between the miR-345 high- and low-expression group [84].

Up to date, neoadjuvant chemoradiotherapy is considered the primary treatment in patients with locally rectal advanced cancer. However, preoperative chemoradiotherapy has not been associated with an OS benefit, which indicates the need for new predictive biomarkers that identify those CRC patients who benefit from this adjuvant therapy [85]. Based on a microarray approach, Yu et al. analyzed the circulating miRNA profile in patients with locally advanced rectal cancer that underwent preoperative chemoradiotherapy (before and after therapy). In this study, the authors found that serum miR-345 expression was significantly downregulated in chemoradiotherapy sensitive patients compared to the chemoradiotherapy-resistant group, showing an AUC of 0.75. According to the survival analysis, low miR-345 expression was associated with superior three-year local recurrence-free survival [86]. In addition, serum miR-125b has also been reported as another predictive biomarker of the preoperative chemoradiotherapy responsiveness in patients with rectal adenocarcinoma. Thus, high serum miR-125b levels identified non-responder from responder patients (AUC $=0.782$ ), observing the same pattern in CRC tissue [87]. 


\subsection{Exosomal miRNAs for CRC Therapy Prediction}

Only two studies have described the potential predictive value of exosomal miRNAs for evaluating the therapeutic efficacy in CRC $[73,88]$. In this study tumor recurrence after FOLFOX treatment in stage III CRC patients was detected in $90 \%$ of the cases with low miR-4772-3p expression levels compared to patients with high expression levels. Although the authors consider that these results need to be validated in a large cohort of patients, their study reveals the potential predictive value of exosomal miRNAs for response to adjuvant chemotherapy. Recently, Jin et al. discovered a panel of 4-serum exosomal miRNAs (miR-21-5p, miR-1246, miR-1229-5p and miR-96-5p) which could discriminate resistant from sensitive CRC patients to conventional chemotherapy with $78 \%$ sensitivity and $88.90 \%$ specificity [88]. These findings provide evidence about the potential of exosomal miRNAs for predicting the resistance to chemotherapy in CRC.

\section{Conclusions and Future Perspectives}

Our review highlighted the potential of circulating miRNAs as novel biomarkers for early detection, diagnosis, prognosis and predictive therapy of CRC. Despite the extensive research in this field, there are still several challenges that remain to be overcome before their clinical application. Several studies have identified associations between circulating miRNA expression patterns and the diagnosis, prognosis and sensitivity to chemotherapy in CRC; however, a further investigation regarding the origin of miRNAs and their biological function is needed. As regulators of gene expression, numerous studies have demonstrated the potential of circulating miRNAs as predictive therapy biomarkers in CRC, however, to elucidate the mechanisms through which miRNAs are involved in the resistance to chemotherapy and other targeted therapies are required. miRNA-based anticancer therapies are being developed, alone or also in combination with other therapies, with the clear objective of overcoming the development of therapy resistance. The option to monitor the status of these miRNAs in liquid biopsies will be a key element in order to apply the anti-miRNA therapeutic strategies during the CRC evolution. Importantly, the mechanisms behind miRNAs alteration in CRC are still quite unknown. To improve the knowledge about these mechanisms would be of great value to design the most accurate miRNAs panel for monitoring CRC patients at different stages of the disease.

In fact, although numerous miRNAs have been examined with the same clinical purpose, there is not a coincidence between single miRNAs or miRNA panels reported by many studies, and even between studies that analyzed the same miRNAs there are sometimes opposite results. These heterogeneous results could be explained by the selection strategy of patients (sample sizes, tumor stages, socio-demographic and clinicopathological characteristics), the samples collection (type of body fluid, collection tubes and protocols), the sample processing and the different approaches for the miRNAs analysis, including data normalization (endogenous or exogenous normalizers) and the use of different cutoff values for the same miRNAs. For example, the harmonization of circulating exosome extraction constitutes a key element to reach a higher reproducibility in studies characterizing exosome-derived miRNAs. Therefore, consensus of the scientific community should be carried out to standardize pre-analytical and post-analytical protocols and to determine the best housekeeping genes to establish a gold standard normalization method. Several studies have demonstrated that cancer-associated circulating miRNAs can be detected in many biofluids, such as urine or saliva. Further studies comparing miRNA profiles in blood and other fluids of the same patient are required to determine the best sample source for a clinical test in CRC patients. In addition, prospective studies including large scale cohorts from multiple centers represent an urgent need to reach an effective clinical implementation of circulating miRNAs-based tests to guide CRC management.

Nowadays, it seems unlikely to use a single miRNA as a diagnostic, prognostic or predictive biomarker for CRC. Several miRNAs have been deregulated in different malignancies, which suggests that miRNAs could not be specific of a cancer type. In this sense, the combination of multiple miRNAs or even the combination of miRNA panels with traditional biomarkers such as CEA or CA19-9 or with clinical variables may provide an increase of the diagnostic accuracy for CRC. Interestingly, 
miRNA panels are based on the combination from two miRNAs up to several miRNAs. However, an ideal clinical model must have both a high sensitivity and specificity and a suitable cost-effectiveness. Although several miRNA-panels for CRC have been reported, until now, cost-benefit analysis has not yet been evaluated. It is necessary to developed cost-benefit models that determine the number of miRNAs that should be included, considering the possible combination with other biomarkers. This approach will allow researchers to know the real possibilities to implement the use of these biomarkers from prevention to treatment management. Thus, this promising strategy will apply a more personalized medicine in CRC.

In conclusion, although future studies must address some important challenges in this field, actually circulating miRNAs could be considered as a molecular mirror of the underlying changes that occur in the development and progression of CRC, providing valuable information on early and advanced diagnosis, prognosis, response to therapy and prediction of treatment response.

Supplementary Materials: The following are available online at http://www.mdpi.com/2072-6694/11/7/898/s1. Table S1: Main findings of the included studies about circulating miRNAs in patients with CRC, Table S2: List of references of Figure 2, Table S3: List of references of Figure 3.

Author Contributions: Conceptualization, M.M.S.-C. and L.M.-R.; writing-original draft preparation, Ó.R.-G., M.M.S.-C. and L.M.-R.; writing-review and editing, A.Á.-C., R.L.-L. and J.I.-C.; supervision, M.M.S.-C and L.M.-R. All authors approved the manuscript.

Funding: This study was supported by the Consellería de Cultura, Educación e Ordenación Universitaria da Xunta de Galicia (Spain), grant number: ED431B2017/029.

Conflicts of Interest: The authors declare no conflicts of interest. The funders had no role in the design of the study; in the collection, analyses, or interpretation of data; in the writing of the manuscript, or in the decision to publish the results.

\section{References}

1. Bray, F.; Ferlay, J.; Soerjomataram, I.; Siegel, R.L.; Torre, L.A.; Jemal, A. Global cancer statistics 2018: GLOBOCAN estimates of incidence and mortality worldwide for 36 cancers in 185 countries. CA Cancer J. Clin. 2018, 68, 394-424. [CrossRef] [PubMed]

2. Arnold, M.; Sierra, M.S.; Laversanne, M.; Soerjomataram, I.; Jemal, A.; Bray, F. Global patterns and trends in colorectal cancer incidence and mortality. Gut 2017, 66, 683-691. [CrossRef] [PubMed]

3. Hughes, L.A.E.; Simons, C.C.J.M.; van den Brandt, P.A.; van Engeland, M.; Weijenberg, M.P. Lifestyle, diet, and colorectal cancer risk according to (Epi)genetic instability: Current evidence and future directions of molecular pathological epidemiology. Curr. Colorectal Cancer Rep. 2017, 13, 455-469. [CrossRef] [PubMed]

4. Ma, H.; Brosens, L.A.A.; Offerhaus, G.J.A.; Giardiello, F.M.; de Leng, W.W.J.; Montgomery, E.A. Pathology and genetics of hereditary colorectal cancer. Pathology 2018, 50, 49-59. [CrossRef] [PubMed]

5. Raskov, H. Colorectal carcinogenesis-update and perspectives. World J. Gastroenterol. 2014, 20, 18151-18164. [CrossRef] [PubMed]

6. Bae, J.M.; Kim, J.H.; Kang, G.H. Molecular subtypes of colorectal cancer and their clinicopathologic features, with an emphasis on the serrated neoplasia pathway. Arch. Pathol. Lab. Med. 2016, 140, 406-412. [CrossRef] [PubMed]

7. Maida, M.; Macaluso, F.S.; Ianiro, G.; Mangiola, F.; Sinagra, E.; Hold, G.; Maida, C.; Cammarota, G.; Gasbarrini, A.; Scarpulla, G. Screening of colorectal cancer: present and future. Expert Rev. Anticancer Ther. 2017, 17, 1131-1146. [CrossRef] [PubMed]

8. Issa, I.A.; Noureddine, M. Colorectal cancer screening: An updated review of the available options. World J. Gastroenterol. 2017, 23, 5086-5096. [CrossRef]

9. Duffy, M.J. Carcinoembryonic antigen as a marker for colorectal cancer: Is it clinically useful? Clin. Chem. 2001, 47, 624-630.

10. Sun, G.; Meng, J.; Duan, H.; Zhang, D.; Tang, Y. Diagnostic assessment of septin9 DNA methylation for colorectal cancer using blood detection: A meta-analysis. Pathol. Oncol. Res. 2018. [CrossRef]

11. Lin, S.; Gregory, R.I. MicroRNA biogenesis pathways in cancer. Nat. Rev. Cancer. 2015, 15, $321-333$. [CrossRef] [PubMed] 
12. Detassis, S.; Grasso, M.; Del Vescovo, V.; Denti, M.A. microRNAs make the call in cancer personalized medicine. Front. Cell Dev. Biol. 2017, 5, 86. [CrossRef] [PubMed]

13. Suzuki, H.I.; Katsura, A.; Matsuyama, H.; Miyazono, K. MicroRNA regulons in tumor microenvironment. Oncogene 2015, 34, 3085-3094. [CrossRef] [PubMed]

14. Heneghan, H.M.; Miller, N.; Kerin, M.J. MiRNAs as biomarkers and therapeutic targets in cancer. Curr. Opin. Pharmacol. 2010, 10, 543-550. [CrossRef] [PubMed]

15. Zhou, K.; Liu, M.; Cao, Y. New insight into microRNA functions in cancer: oncogene-microRNA-tumor suppressor gene network. Front. Mol. Biosci. 2017, 4, 46. [CrossRef]

16. Shin, V.Y.; Ng, E.K.; Chan, V.W.; Kwong, A.; Chu, K.M. A three-miRNA signature as promising non-invasive diagnostic marker for gastric cancer. Mol. Cancer 2015, 14, 202. [CrossRef]

17. Matamala, N.; Vargas, M.T.; González-Cámpora, R.; Miñambres, R.; Arias, J.I.; Menéndez, P.; Andrés-León, E.; Gómez-López, G.; Yanowsky, K.; Calvete-Candenas, J.; et al. Tumor microRNA expression profiling identifies circulating microRNAs for early breast cancer detection. Clin. Chem. 2015, 61, 1098-1106. [CrossRef]

18. Kobayashi, M.; Sawada, K.; Nakamura, K.; Yoshimura, A.; Miyamoto, M.; Shimizu, A.; Ishida, K.; Nakatsuka, E.; Kodama, M.; Hashimoto, K.; et al. Exosomal miR-1290 is a potential biomarker of high-grade serous ovarian carcinoma and can discriminate patients from those with malignancies of other histological types. J. Ovarian Res. 2018, 11, 81. [CrossRef]

19. Weber, J.A.; Baxter, D.H.; Zhang, S.; Huang, D.Y.; How Huang, K.; Jen Lee, M.; Galas, D.J.; Wang, K. The microRNA spectrum in 12 body fluids. Clin. Chem. 2010, 56, 1733-1741. [CrossRef]

20. Matsuzaki, J.; Ochiya, T. Circulating microRNAs and extracellular vesicles as potential cancer biomarkers: a systematic review. Int. J. Clin. Oncol. 2017, 22, 413-420. [CrossRef]

21. Zen, K.; Zhang, C.Y. Circulating microRNAs: A novel class of biomarkers to diagnose and monitor human cancers. Med. Res. Rev. 2012, 32, 326-348. [CrossRef] [PubMed]

22. Ng, E.K.; Chong, W.W.; Jin, H.; Lam, E.K.; Shin, V.Y.; Yu, J.; Poon, T.C.; Ng, S.S.; Sung, J.J. Differential expression of microRNAs in plasma of patients with colorectal cancer: a potential marker for colorectal cancer screening. Gut 2009, 58, 1375-1381. [CrossRef] [PubMed]

23. Zekri, A.R.; Youssef, A.S.; Lotfy, M.M.; Gabr, R.; Ahmed, O.S.; Nassar, A.; Hussein, N.; Omran, D.; Medhat, E.; Eid, S.; et al. Circulating serum miRNAs as diagnostic markers for colorectal cancer. PLoS ONE 2016, 11, e0154130. [CrossRef] [PubMed]

24. Pan, C.; Yan, X.; Li, H.; Huang, L.; Yin, M.; Yang, Y.; Gao, R.; Hong, L.; Ma, Y.; Shi, C.; et al. Systematic literature review and clinical validation of circulating microRNAs as diagnostic biomarkers for colorectal cancer. Oncotarget 2017, 8, 68317-68328. [CrossRef] [PubMed]

25. Huang, Z.; Huang, D.; Ni, S.; Peng, Z.; Sheng, W.; Du, X. Plasma microRNAs are promising novel biomarkers for early detection of colorectal cancer. Int. J. Cancer 2010, 127, 118-126. [CrossRef] [PubMed]

26. Wang, Q.; Huang, Z.; Ni, S.; Xiao, X.; Xu, Q.; Wang, L.; Huang, D.; Tan, C.; Sheng, W.; Du, X. Plasma miR-601 and miR-760 are novel biomarkers for the early detection of colorectal cancer. PLOS ONE 2012, 7, e44398. [CrossRef] [PubMed]

27. Yamada, A.; Horimatsu, T.; Okugawa, Y.; Nishida, N.; Honjo, H.; Ida, H.; Kou, T.; Kusaka, T.; Sasaki, Y.; Yagi, M.; et al. Serum miR-21, miR-29a, and miR-125b are promising biomarkers for the early detection of colorectal neoplasia. Clin. Cancer Res. 2015, 21, 4234-4242. [CrossRef]

28. Wang, S.; Xiang, J.; Li, Z.; Lu, S.; Hu, J.; Gao, X.; Yu, L.; Wang, L.; Wang, J.; Wu, Y.; et al. A plasma microRNA panel for early detection of colorectal cancer. Int. J. Cancer 2015, 136, 152-161. [CrossRef]

29. Kanaan, Z.; Roberts, H.; Eichenberger, M.R.; Billeter, A.; Ocheretner, G.; Pan, J.; Rai, S.N.; Jorden, J.; Williford, A.; Galandiuk, S. A plasma microRNA panel for detection of colorectal adenomas: a step toward more precise screening for colorectal cancer. Ann. Surg. 2013, 258, 400-408. [CrossRef]

30. Hofsli, E.; Sjursen, W.; Prestvik, W.S.; Johansen, J.; Rye, M.; Tranø, G.; Wasmuth, H.H.; Hatlevoll, I.; Thommesen, L. Identification of serum microRNA profiles in colon cancer. Br. J. Cancer 2013, 108, 1712-1719. [CrossRef]

31. Vychytilova-Faltejskova, P.; Radova, L.; Sachlova, M.; Kosarova, Z.; Slaba, K.; Fabian, P.; Grolich, T.; Prochazka, V.; Kala, Z.; Svoboda, M.; et al. Serum-based microRNA signatures in early diagnosis and prognosis prediction of colon cancer. Carcinogenesis 2016, 37, 941-950. [CrossRef] [PubMed] 
32. Herreros-Villanueva, M.; Duran-Sanchon, S.; Martín, A.C.; Pérez-Palacios, R.; Vila-Navarro, E.; Marcuello, M.; Diaz-Centeno, M.; Cubiella, J.; Diez, M.S.; Bujanda, L.; et al. Plasma microRNA signature validation for early detection of colorectal cancer. Clin. Transl. Gastroenterol. 2019, 10, e00003. [CrossRef] [PubMed]

33. Liu, X.; Xu, T.; Hu, X.; Chen, X.; Zeng, K.; Sun, L.; Wang, S. Elevated circulating miR-182 acts as a diagnostic biomarker for early colorectal cancer. Cancer Manag. Res. 2018, 10, 857-865. [CrossRef] [PubMed]

34. Krawczyk, P.; Powrózek, T.; Olesiński, T.; Dmitruk, A.; Dziwota, J.; Kowalski, D.; Milanowski, J. Evaluation of miR-506 and miR-4316 expression in early and non-invasive diagnosis of colorectal cancer. Int. J. Colorectal Dis. 2017, 32, 1057-1060. [CrossRef] [PubMed]

35. Sun, Y.; Yang, B.; Lin, M.; Yu, H.; Chen, H.; Zhang, Z. Identification of serum miR-30a-5p as a diagnostic and prognostic biomarker in colorectal cancer. Cancer Biomark. 2019, 24, 299-305. [CrossRef] [PubMed]

36. Cekaite, L.; Eide, P.W.; Lind, G.E.; Skotheim, R.I. MicroRNAs as growth regulators, their function and biomarker status in colorectal cancer. Oncotarget 2015, 7, 6476-6505. [CrossRef] [PubMed]

37. Cheng, H.; Zhang, L.; Cogdell, D.E.; Zheng, H.; Schetter, A.J.; Nykter, M.; Harris, C.C.; Chen, K.; Hamilton, S.R.; Zhang, W. Circulating plasma miR-141 is a novel biomarker for metastatic colon cancer and predicts poor prognosis. PLoS ONE 2011, 6, e17745. [CrossRef]

38. Wang, L.G.; Gu, J. Serum microRNA-29a is a promising novel marker for early detection of colorectal liver metastasis. Cancer Epidemiol. 2012, 36, e61-e67. [CrossRef]

39. Ding, M.; Zhang, T.; Li, S.; Zhang, Y.; Qiu, Y.; Zhang, B. Correlation analysis between liver metastasis and serum levels of miR-200 and miR-141 in patients with colorectal cancer. Mol. Med. Rep. 2017, 16, 7791-7795. [CrossRef]

40. Yin, J.; Bai, Z.; Song, J.; Yang, Y.; Wang, J.; Han, W.; Zhang, J.; Meng, H.; Ma, X.; Yang, Y.; et al. Differential expression of serum miR-126, miR-141 and miR-21 as novel biomarkers for early detection of liver metastasis in colorectal cancer. Chin. J. Cancer Res. 2014, 26, 95-103.

41. Zhu, S.H.; He, X.C.; Wang, L. Correlation analysis of miR-200b, miR-200c, and miR-141 with liver metastases in colorectal cancer patients. Eur. Rev. Med. Pharmacol. Sci. 2017, 21, 2357-2363. [PubMed]

42. Hur, K.; Toiyama, Y.; Schetter, A.J.; Okugawa, Y.; Harris, C.C.; Boland, C.R.; Goel, A. Identification of a metastasis-specific microRNA signature in human colorectal cancer. J. Natl. Cancer Inst. 2015, 107. [CrossRef] [PubMed]

43. Wang, D.S.; Zhong, B.; Zhang, M.S.; Gao, Y. Upregulation of serum miR-103 predicts unfavorable prognosis in patients with colorectal cancer. Eur. Rev. Med. Pharmacol. Sci. 2018, 22, 4518-4523. [PubMed]

44. Giráldez, M.D.; Lozano, J.J.; Ramírez, G.; Hijona, E.; Bujanda, L.; Castells, A.; Gironella, M. Circulating microRNAs as biomarkers of colorectal cancer: Results from a genome-wide profiling and validation study. Clin. Gastroenterol. Hepatol. 2013, 11, 681-688. [CrossRef] [PubMed]

45. Brunet Vega, A.; Pericay, C.; Moya, I.; Ferrer, A.; Dotor, E.; Pisa, A.; Casalots, À.; Serra-Aracil, X.; Oliva, J.C.; Ruiz, A.; et al. microRNA expression profile in stage III colorectal cancer: Circulating miR-18a and miR-29a as promising biomarkers. Oncol. Rep. 2013, 30, 320-326. [CrossRef] [PubMed]

46. Sun, Y.; Liu, Y.; Cogdell, D.; Calin, G.A.; Sun, B.; Kopetz, S.; Hamilton, S.R.; Zhang, W. Examining plasma microRNA markers for colorectal cancer at different stages. Oncotarget 2016, 7, 11434-11449. [CrossRef] [PubMed]

47. Ng, L.; Wan, T.M.; Man, J.H.; Chow, A.K.; Iyer, D.; Chen, G.; Yau, T.C.; Lo, O.S.; Foo, D.C.; Poon, J.T.; et al. Identification of serum miR-139-3p as a non-invasive biomarker for colorectal cancer. Oncotarget 2017, 8, 27393-27400. [CrossRef] [PubMed]

48. Ogata-Kawata, H.; Izumiya, M.; Kurioka, D.; Honma, Y.; Yamada, Y.; Furuta, K.; Gunji, T.; Ohta, H.; Okamoto, H.; Sonoda, H.; et al. Circulating exosomal microRNAs as biomarkers of colon cancer. PLoS ONE 2014, 9, e92921. [CrossRef] [PubMed]

49. Wang, J.; Yan, F.; Zhao, Q.; Zhan, F.; Wang, R.; Wang, L.; Zhang, Y.; Huang, X. Circulating exosomal miR-125a-3p as a novel biomarker for early-stage colon cancer. Sci. Rep. 2017, 7, 4150. [CrossRef]

50. Tsukamoto, M.; Iinuma, H.; Yagi, T.; Matsuda, K.; Hashiguchi, Y. Circulating exosomal microRNA-21 as a biomarker in each tumor stage of colorectal cancer. Oncology 2017, 92, 360-370. [CrossRef]

51. Yan, S.; Han, B.; Gao, S.; Wang, X.; Wang, Z.; Wang, F.; Zhang, J.; Xu, D.; Sun, B. Exosome-encapsulated microRNAs as circulating biomarkers for colorectal cancer. Oncotarget 2017, 8, 60149-60158. [CrossRef] [PubMed] 
52. Zhang, H.; Zhu, M.; Shan, X.; Zhou, X.; Wang, T.; Zhang, J.; Tao, J.; Cheng, W.; Chen, G.; Li, J.; et al. A panel of seven-miRNA signature in plasma as potential biomarker for colorectal cancer diagnosis. Gene 2019, 687, 246-254. [CrossRef] [PubMed]

53. Liu, X.; Pan, B.; Sun, L.; Chen, X.; Zeng, K.; Hu, X.; Xu, T.; Xu, M.; Wang, S. Circulating exosomal miR-27a and miR-130a act as novel diagnostic and prognostic biomarkers of colorectal cancer. Cancer Epidemiol. Biomark. Prev. 2018, 27, 746-754. [CrossRef]

54. Pu, X.; Huang, G.; Guo, H.; Guo, C.; Li, H.; Ye, S.; Ling, S.; Jiang, L.; Tian, Y.; Lin, T. Circulating miR-221 directly amplified from plasma is a potential diagnostic and prognostic marker of colorectal cancer and is correlated with p53 expression. J. Gastroenterol. Hepatol. 2010, 25, 1674-1680. [CrossRef] [PubMed]

55. Li, J.; Liu, Y.; Wang, C.; Deng, T.; Liang, H.; Wang, Y.; Huang, D.; Fan, Q.; Wang, X.; Ning, T.; et al. Serum miRNA expression profile as a prognostic biomarker of stage II/III colorectal adenocarcinoma. Sci. Rep. 2015, 5, 12921. [CrossRef] [PubMed]

56. Toiyama, Y.; Takahashi, M.; Hur, K.; Nagasaka, T.; Tanaka, K.; Inoue, Y.; Kusunoki, M.; Boland, C.R.; Goel, A. Serum miR-21 as a diagnostic and prognostic biomarker in colorectal cancer. J. Natl. Cancer Inst. 2013, 105, 849-859. [CrossRef] [PubMed]

57. Liu, G.H.; Zhou, Z.G.; Chen, R.; Wang, M.J.; Zhou, B.; Li, Y.; Sun, X.F. Serum miR-21 and miR-92a as biomarkers in the diagnosis and prognosis of colorectal cancer. Tumor Biol. 2013, 34, 2175-2181. [CrossRef]

58. Imaoka, H.; Toiyama, Y.; Fujikawa, H.; Hiro, J.; Saigusa, S.; Tanaka, K.; Inoue, Y.; Mohri, Y.; Mori, T.; Kato, T.; et al. Circulating microRNA-1290 as a novel diagnostic and prognostic biomarker in human colorectal cancer. Ann. Oncol. 2016, 27, 1879-1886. [CrossRef]

59. Wang, W.; Qu, A.; Liu, W.; Liu, Y.; Zheng, G.; Du, L.; Zhang, X.; Yang, Y.; Wang, C.; Chen, X. Circulating miR-210 as a diagnostic and prognostic biomarker for colorectal cancer. Eur. J. Cancer Care 2017, 26. [CrossRef]

60. Yuan, D.; Li, K.; Zhu, K.; Yan, R.; Dang, C. Plasma miR-183 predicts recurrence and prognosis in patients with colorectal cancer. Cancer Biol. Ther. 2015, 16, 268-275. [CrossRef]

61. Liu, M.; Zhi, Q.; Wang, W.; Zhang, Q.; Fang, T.; Ma, Q. Up-regulation of miR-592 correlates with tumor progression and poor prognosis in patients with colorectal cancer. Biomed. Pharmacother. 2015, 69, $214-220$. [CrossRef] [PubMed]

62. Xu, C.; Gu, L. The diagnostic effect of serum miR-196b as biomarker in colorectal cancer. Biomed. Rep. 2017, 6, 39-45. [CrossRef] [PubMed]

63. Lv, Z.; Fan, Y.; Chen, H.; Zhao, D. Investigation of microRNA-155 as a serum diagnostic and prognostic biomarker for colorectal cancer. Tumor Biol. 2015, 36, 1619-1625. [CrossRef]

64. Hur, K.; Toiyama, Y.; Okugawa, Y.; Ide, S.; Imaoka, H.; Boland, C.R.; Goel, A. Circulating microRNA-203 predicts prognosis and metastasis in human colorectal cancer. Gut 2017, 66, 654-665. [CrossRef] [PubMed]

65. Maierthaler, M.; Benner, A.; Hoffmeister, M.; Surowy, H.; Jansen, L.; Knebel, P.; Chang-Claude, J.; Brenner, H.; Burwinkel, B. Plasma miR-122 and miR-200 family are prognostic markers in colorectal cancer. Int. J. Cancer 2017, 140, 176-187. [CrossRef] [PubMed]

66. Tayel, S.I.; Fouda, E.A.M.; Gohar, S.F.; Elshayeb, E.I.; El-sayed, E.H.; El-kousy, S.M. Potential role of microRNA 200c gene expression in assessment of colorectal cancer. Arch. Biochem. Biophys. 2018, 647, 41-46. [CrossRef] [PubMed]

67. Toiyama, Y.; Hur, K.; Tanaka, K.; Inoue, Y.; Kusunoki, M.; Boland, C.R.; Goel, A. Serum miR-200c is a novel prognostic and metastasis-predictive biomarker in patients with colorectal cancer. Ann. Surg. 2014, 259, 735-743. [CrossRef]

68. Santasusagna, S.; Moreno, I.; Navarro, A.; Martinez Rodenas, F.; Hernández, R.; Castellano, J.J.; Muñoz, C.; Monzo, M. Prognostic impact of miR-200 family members in plasma and exosomes from tumor-draining versus peripheral veins of colon cancer patients. Oncology 2018, 95, 309-318. [CrossRef]

69. Basati, G.; Razavi, A.E.; Pakzad, I.; Malayeri, F.A. Circulating levels of the miRNAs, miR-194, and miR-29b, as clinically useful biomarkers for colorectal cancer. Tumor Biol. 2016, 37, 1781-1788. [CrossRef]

70. Kou, C.H.; Zhou, T.; Han, X.L.; Zhuang, H.J.; Qian, H.X. Downregulation of mir-23b in plasma is associated with poor prognosis in patients with colorectal cancer. Oncol. Lett. 2016, 12, 4838-4844. [CrossRef]

71. Vafaee, F.; Diakos, C.; Kirschner, M.B.; Reid, G.; Michael, M.Z.; Horvath, L.G.; Alinejad-Rokny, H.; Cheng, Z.J.; Kuncic, Z.; Clarke, S. A data-driven, knowledge-based approach to biomarker discovery: application to circulating microRNA markers of colorectal cancer prognosis. NPJ Syst. Biol. Appl. 2018, 4, 20. [CrossRef] [PubMed] 
72. Matsumura, T.; Sugimachi, K.; Iinuma, H.; Takahashi, Y.; Kurashige, J.; Sawada, G.; Ueda, M.; Uchi, R.; Ueo, H.; Takano, Y.; et al. Exosomal microRNA in serum is a novel biomarker of recurrence in human colorectal cancer. Br. J. Cancer 2015, 113, 275-281. [CrossRef] [PubMed]

73. Liu, C.; Eng, C.; Shen, J.; Lu, Y.; Takata, Y.; Mehdizadeh, A.; Chang, G.J.; Rodriguez-Bigas, M.A.; Li, Y.; Chang, P.; et al. Serum exosomal miR-4772-3p is a predictor of tumor recurrence in stage II and III colon cancer. Oncotarget 2016, 7, 76250-76260. [CrossRef] [PubMed]

74. Yan, S.; Jiang, Y.; Liang, C.; Cheng, M.; Jin, C.; Duan, Q.; Xu, D.; Yang, L.; Zhang, X.; Ren, B.; et al. Exosomal miR-6803-5p as potential diagnostic and prognostic marker in colorectal cancer. J. Cell. Biochem. 2018, 119, 4113-4119. [CrossRef] [PubMed]

75. Peng, Z.; Gu, R.; Yan, B. Downregulation of exosome-encapsulated miR-548c-5p is associated with poor prognosis in colorectal cancer. J. Cell. Biochem. 2019, 120, 1457-1463. [CrossRef]

76. Fu, F.; Jiang, W.; Zhou, L.; Chen, Z. Circulating exosomal miR-17-5p and miR-92a-3p predict pathologic stage and grade of colorectal cancer. Transl. Oncol. 2018, 11, 221-232. [CrossRef] [PubMed]

77. Chen, Q.; Xia, H.W.; Ge, X.J.; Zhang, Y.C.; Tang, Q.L.; Bi, F. Serum miR-19a predicts resistance to FOLFOX chemotherapy in advanced colorectal cancer cases. Asian Pac. J. Cancer Prev. 2013, 14, 7421-7426. [CrossRef] [PubMed]

78. Zhang, J.; Zhang, K.; Bi, M.; Jiao, X.; Zhang, D.; Dong, Q. Circulating microRNA expressions in colorectal cancer as predictors of response to chemotherapy. Anti-Cancer Drugs 2014, 25, 346-352. [CrossRef]

79. Kjersem, J.B.; Ikdahl, T.; Lingjaerde, O.C.; Guren, T.; Tveit, K.M.; Kure, E.H. Plasma microRNAs predicting clinical outcome in metastatic colorectal cancer patients receiving first-line oxaliplatin-based treatment. Mol. Oncol. 2014, 8, 59-67. [CrossRef]

80. Ji, D.; Qiao, M.; Yao, Y.; Li, M.; Chen, H.; Dong, Q.; Jia, J.; Cui, X.; Li, Z.; Xia, J.; et al. Serum-based microRNA signature predicts relapse and therapeutic outcome of adjuvant chemotherapy in colorectal cancer patients. EBioMedicine 2018, 35, 189-197. [CrossRef]

81. Zeng, Z.; Li, Y.; Pan, Y.; Lan, X.; Song, F.; Sun, J.; Zhou, K.; Liu, X.; Ren, X.; Wang, F.; et al. Cancer-derived exosomal miR-25-3p promotes pre-metastatic niche formation by inducing vascular permeability and angiogenesis. Nat. Commun. 2018, 9, 5395. [CrossRef] [PubMed]

82. Hansen, T.F.; Carlsen, A.L.; Heegaard, N.H.H.; Sørensen, F.B.; Jakobsen, A. Changes in circulating microRNA-126 during treatment with chemotherapy and bevacizumab predicts treatment response in patients with metastatic colorectal cancer. Br. J. Cancer 2015, 112, 624-629. [CrossRef] [PubMed]

83. Ulivi, P.; Canale, M.; Passardi, A.; Marisi, G.; Valgiusti, M.; Frassineti, G.; Calistri, D.; Amadori, D.; Scarpi, E. Circulating plasma levels of miR-20b, miR-29b and miR-155 as predictors of bevacizumab efficacy in patients with metastatic colorectal cancer. Int. J. Mol. Sci. 2018, 19, 307. [CrossRef] [PubMed]

84. Schou, J.V.; Rossi, S.; Jensen, B.V.; Nielsen, D.L.; Pfeiffer, P.; Høgdall, E.; Yilmaz, M.; Tejpar, S.; Delorenzi, M.; Kruhøffer, M.; et al. miR-345 in metastatic colorectal cancer: A non-invasive biomarker for clinical outcome in non-KRAS mutant patients treated with 3rd line cetuximab and irinotecan. PLoS ONE 2014, 9, e99886. [CrossRef] [PubMed]

85. Brændengen, M.; Glimelius, B. Preoperative radiotherapy or chemoradiotherapy in rectal cancer - Is survival improved? An update of the "Nordic" LARC study in non-resectable cancers. Radiother. Oncol. 2018, 127, 392-395. [CrossRef] [PubMed]

86. Yu, J.; Li, N.; Wang, X.; Ren, H.; Wang, W.; Wang, S.; Song, Y.; Liu, Y.; Li, Y.; Zhou, X.; et al. Circulating serum microRNA-345 correlates with unfavorable pathological response to preoperative chemoradiotherapy in locally advanced rectal cancer. Oncotarget 2016, 7, 64233-64243. [CrossRef] [PubMed]

87. D’Angelo, E.; Fassan, M.; Maretto, I.; Pucciarelli, S.; Zanon, C.; Digito, M.; Rugge, M.; Nitti, D.; Agostini, M. Serum miR-125b is a non-invasive predictive biomarker of the pre-operative chemoradiotherapy responsiveness in patients with rectal adenocarcinoma. Oncotarget 2016, 7, 28647-28657. [CrossRef]

88. Jin, G.; Liu, Y.; Zhang, J.; Bian, Z.; Yao, S.; Fei, B.; Zhou, L.; Yin, Y.; Huang, Z. A panel of serum exosomal microRNAs as predictive markers for chemoresistance in advanced colorectal cancer. Cancer Chemother. Pharmacol. 2019. [CrossRef]

(C) 2019 by the authors. Licensee MDPI, Basel, Switzerland. This article is an open access article distributed under the terms and conditions of the Creative Commons Attribution (CC BY) license (http://creativecommons.org/licenses/by/4.0/). 